\title{
TECHNICAL ADVANTAGES AND DISADVANTAGES OF BIARTICULAR ACTUATORS IN BIPEDAL ROBOTS
}

\author{
Atabak Nejadfard and Karsten Berns and Patrick Vonwirth \\ Robotics Research LAB, Department of Computer Science, \\ Technical University of Kaiserslautern, \\ Gottlieb-Daimler-Str. Building 48, 67663 Kaiserslautern, Germany \\ E-mail: nejadfard@cs.uni-kl.de,berns@cs.uni-kl.de,vonwirth@cs.uni-kl.de
}

\begin{abstract}
The practical advantages of redundant biarticular actuators in bipedal robotic systems are demonstrated and reviewed. The major physiological role of biarticular muscles indeed can be successfully replicated in robotic systems by transferring power from heavy upper leg segments to the lower limb joints. As hypothesized in biomechanics, biarticular actuators contribute to the efficiency of explosive movements in robotic systems similar to the human body. Despite the added weight of the redundant actuators, redundancy enables the proper selection of mono-articular actuators so that all the actuators operate in their optimum working points. It is important to notice that the energetic benefits of biarticular muscles have not been identified during human walking locomotion. Therefore the efficiency gain due to the addition of biarticulars can be limited in bipedal walking. Still, the technical advantage of the bi-articulation facilitates the mass distribution of the actuators over leg segments, specifically by reducing the mass of the shank segment in the robotic leg.
\end{abstract}

\section{Introduction}

Motor redundancy in the form of biarticular muscles (BIMs) contributes to the efficiency of human locomotion. The growing number of researchers are utilizing biarticular actuators (BIAs) in designing exoskeletons and bipedal robots 1 We have designed and constructed Compliant robotic leg (CARL) based on the physiology of the human leg muscles. Numerous studies have iterated the advantages of BIMs in human locomotion and advocated their application in robotic platforms. In this paper, we discuss the advantages and disadvantages of BiAs from a technical perspective. CARL is used as a benchmark to illustrate the arising challenges when exploiting the advantages of bi-articulation.

BIMs span two joints and when activated drive simultaneously either joints in contrast to the standard mono-articular muscles (MoMs) which span only one joint. These muscles are highly utilized in the locomotive limbs of humans and animals. 2 The major BIMs in the human leg are Hamstrings (HAM), Rectus Femoris (RF), and Gastrocnemius (GA). The muscular structure of the human leg is illustrated in Fig. 1d. It is hypothesized that BIMs in a human act similar to ligaments, experiencing relatively low contraction velocity while transferring power from heavier upper limb muscles to the lower limb joints.

BIAs are investigated in three robotic domains: manipulators, exoskeletons and musculoskeletal robots. It is shown in manipulators that by adding redundant BIAs, the end-effector force of the manipulator can be increased; furthermore, the maximum force polygon around the end-effector would become uniform relative to the conventional manipulators 3 The manipulator would be able to generate consistent force in all the directions resulting in an adept impedance control.

The major application of BIAs is in Musculoskeletal robots (MSkRs) 4 These robots are mostly constructed as a test platform to mimic the musculature of the human body to transfer 


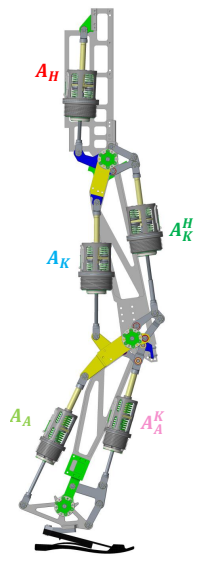

(a)

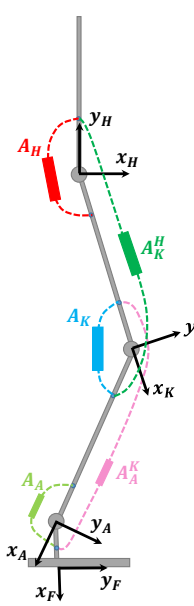

(b)

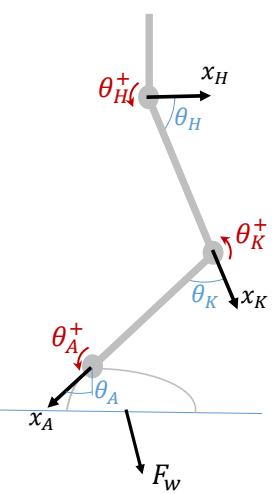

(c)

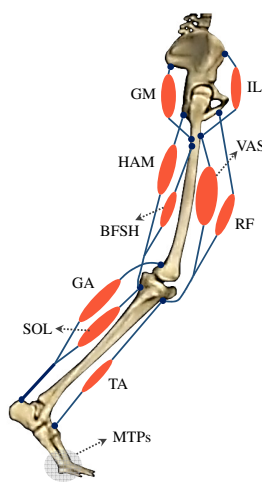

(d)

Fig. 1. (a) The CAD model of the planar Compliant robotic leg (CARL) and (b) the connection network of the actuators. CARL has 5 linear Series elastic actuators (SEAs). Three actuators in the backside of the leg are mono-articular. The two frontal actuators are the biarticular actuators. (c) The definition of the medical joint angles is illustrated which is used for calculating the moment arm values. (d) The abstraction of the human leg major muscles generation movements along the sagittal plane. The insertion/origin sites of the muscles are inaccurately depicted for purpose of better visualization.

the biological efficacy of the human body to the robotic leg. Due to the complex structure, it is not possible to have a precise force and position control and therefore MSkRs are only capable of performing basic movements like hopping and jumping which require limited feedback control, unlike conventional bipedal robots 415

We have designed CARL to bridge the gap between conventional and musculoskeletal bipedal robots. Series elastic actuators (SEAs) are used to provide precise force and position control in joints 6 6 67 Besides, CARL features two BIAs, to partly replicate the motor redundancy of the human leg. The energetics of actuators can be investigated specifically in case of biarticulars since the superior mechatronics of CARL allows us to accurately measure power, force and velocity of the actuators and angular velocity and torque in the joints. Consequently, the biomechanical hypothesis can be evaluated using CARL.

In this paper, we analyze the action of the BIAs and investigate the technical challenges and deviations from expected biological roles. Our analysis is highly dependent on human anatomic and locomotion data. The objective of this paper is to address the question that whether the addition of redundant BiAs can be justified for different locomotion types.

\section{Design of Compliant Robotic Leg (Carl)}

The planar robotic leg CARL is designed based on the human morphology 899 CARL consists of three conventional mono-articular actuators (MoAs), denoted as $A_{H}, A_{K}$ and $A_{A}$, acting on the hip, knee and ankle joints, respectively, see Fig. 1b. Additionally, there are two redundant BiAs, $A_{K}^{H}$, and $A_{A}^{K}$. The $A_{K}^{H}$ is spanning the hip and the knee joints, reproducing the action of HAM and RF muscles. The biarticular $A_{A}^{K}$ is spanning the knee and the ankle joints similar to the Gastrocnemius (GA) muscle. The thigh and shank links have equal length of $42 \mathrm{~cm}$ with a mass of respectively $7.5 \mathrm{~kg}$ and $3.5 \mathrm{~kg}$, comparable to the human leg morphology. A prosthetic foot is 


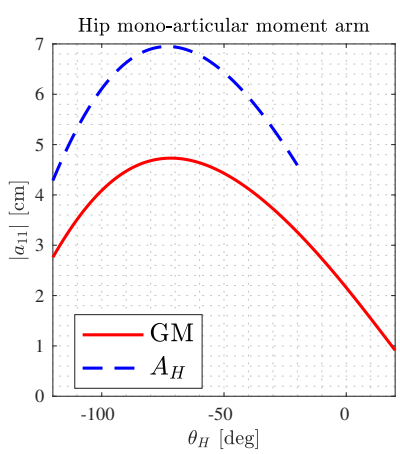

(a)

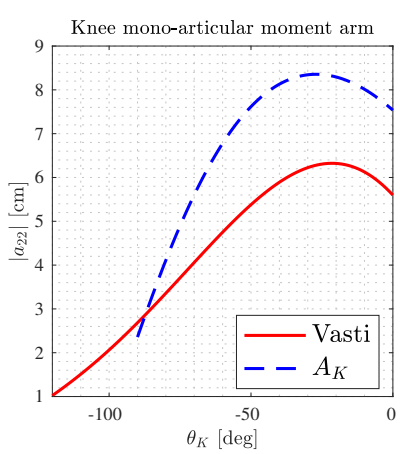

(b)

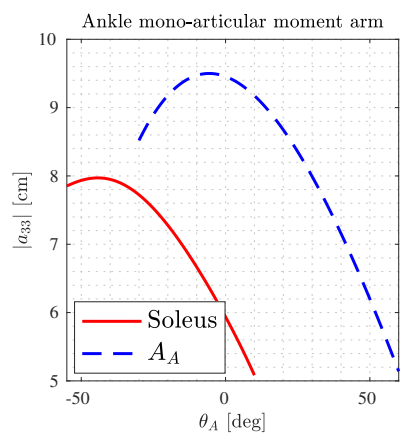

(c)

Fig. 2. The moment arm profiles of the mono-articular actuator (MoA) in (a) hip, (b) knee, and (c) ankle joints. The Moment Arm Profile (MAP) of corresponding muscles are shown in red. These muscles are Gluteus Maximus (GM), Vasti muscle group (VAS) and Soleus (SOL), respectively at hip, knee and ankle.

used to provide the highest resemblance in behavior and size to the human foot.

\subsection{Actuation kinematics}

One of the major challenges of adding a redundant actuator is to determine its interaction with other existing actuators. The severity of the problem increases with adding two redundant actuator as in CARL. In order to investigate the interaction of the actuators with each other, we introduce the moment arm matrix $\boldsymbol{A}_{m}$ which essentially defines the actuator kinematics. $\boldsymbol{A}_{\boldsymbol{m}}$ provides a mapping from the joint space to the muscle space, where

$$
\begin{aligned}
\boldsymbol{V}_{\boldsymbol{m}} & =\boldsymbol{A}_{\boldsymbol{m}} \boldsymbol{\omega}, \\
\boldsymbol{\tau} & =\boldsymbol{A}_{\boldsymbol{m}}^{\boldsymbol{T}} \boldsymbol{F}_{\boldsymbol{m}} .
\end{aligned}
$$

In which $\boldsymbol{\omega}$ and $\boldsymbol{\tau}$ are the angular velocity and the torque vectors in the joint space. The actuator force vector is defined as $\boldsymbol{F}_{\boldsymbol{m}}=\left[F_{H}, F_{K}, F_{A}, F_{K}^{H}, F_{A}^{K}\right]^{T}$. Note that the elements of the vector belong to the actuators with identical indices. The muscle velocity vector $\boldsymbol{V}_{\boldsymbol{m}}$ is defined similarly to the force vector. Extension force and velocity define the positive direction for the actuators. The positive direction for the joint torques and the angular velocities are presented in Fig. 1c.

In CARL, with five muscles and three joints, the moment arm matrix is defined as

$$
\boldsymbol{A}_{\boldsymbol{m}}(\boldsymbol{\theta})=\left[\begin{array}{ccccc}
a_{11} & 0 & 0 & -a_{14} & 0 \\
0 & a_{22} & 0 & -a_{24} & -a_{25} \\
0 & 0 & a_{33} & 0 & -a_{35}
\end{array}\right]^{T}
$$

Where $\boldsymbol{\theta}=\left[\theta_{H}, \theta_{K}, \theta_{A}\right]^{T}$ is the vector of joint angles. $a_{11}\left(\theta_{H}\right), a_{22}\left(\theta_{K}\right), a_{33}\left(\theta_{A}\right)$ are the lever arms of the MoAs which exert positive moments on the joints during extension. $a_{14}\left(\theta_{H}, \theta_{K}\right)$ and $a_{24}\left(\theta_{H}, \theta_{K}\right)$ are the lever arms of the biarticular $A_{K}^{H}$, acting on the hip and the knee joints, respectively. Similarly, the lever arms of the biarticular $A_{A}^{K}$ are $a_{25}\left(\theta_{K}, \theta_{A}\right)$ and $a_{35}\left(\theta_{K}, \theta_{A}\right)$, connected to the knee and the ankle joints.

\subsection{Design criteria}

The selection of the moment arms, components of $\boldsymbol{A}_{\boldsymbol{m}}$, is critical to attaining an efficient actuation network. To illustrate the interconnection of the actuators, consider that it is desired to generate 

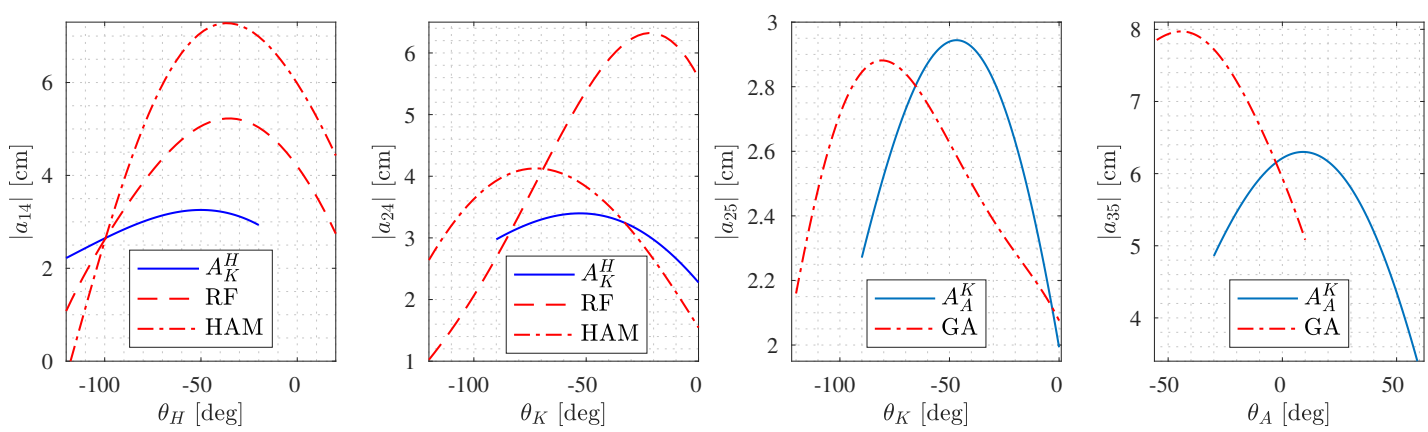

Fig. 3. The moment arm profiles of biarticular actuators compared with corresponding human leg muscles.

a flexing torque at the knee. There are three actuators $\left(A_{K}, A_{K}^{H}, A_{A}^{K}\right)$ that simultaneously act on the knee joint. The force on these actuators has to be calculated in such a way that the desired torque can be achieved at the knee. However, the activation of biarticulars $A_{K}^{H}$ and $A_{A}^{K}$ would yield secondary moments at hip and ankle joints, respectively. The moments at the hip and ankle should also be compensated by mono-articulars at these joints. Eventually, all the actuators would be activated to generate a flexing motion at the knee.

The design paradigm of actuation network is presented in Ref. 910. In linear actuation system, as in skeletal muscle, the moment arm is not constant but is a function of the joint angles which introduces a nonlinear relationship between actuator/muscle force and its resultant moment at the joint. In this article, the nonlinear curve is called Moment Arm Profile (MAP). The design principle is based on replicating the MAP of the human leg muscles for MoAs. The assumption is that the musculature anatomy is optimized to achieve an efficient locomotion performance.

The MAP for biarticular actuators is more complicated to adopt since it is dependent on two joint angles and can not be exactly copied with the mechanical construction of the robot. The determining design factor is adopted from the biomechanical notion of muscle principal direction introduced in Ref. 11. The principal direction of an individual muscle is defined as the direction of the force at the ground contact point produced by the same muscle assuming that all the other muscles are inactivated. Indeed, the principal direction of one muscle determines its directional contribution to the ground reaction force. By replicating the principal direction of each muscle it is guaranteed that the functional role of muscle would be reproduced in the robotic leg.

The principal direction of mono-articular muscles is aligned by the non-connecting link segment, depending on the posture of the leg. For instance, the principal direction of $A_{H}$ is parallel to the direction of the shank link. On the contrary, the principal direction of the biarticulars is dependent on the Ratio of Moment Arms (RMA) at both ends of the muscle $\frac{10}{2}$ regardless of the leg posture. RMA provides a simplified criterion for selecting actuation kinematics of a biarticular actuator as RMA is fairly constant for human muscles in diverse locomotion types.

Moment arm profiles of the MOAs in CARL is constructed with a similar curvature of the corresponding leg muscles, see Fig. 2. The only exception is the ankle joint where the profile is shifted toward positive angles. Due to the lack of Metatarsophalangeal Joints (MTP) in the prosthetic foot of CARL, the ankle joint in the robot should have a higher flexing range than the human ankle, which explains the angular shift in MAP of the $A_{A}$ actuator. The maximum moment arm value for each MoA is higher in the robot relative to its biological counterpart, mainly because the actuators generate maximum force much lower than muscles and therefore 


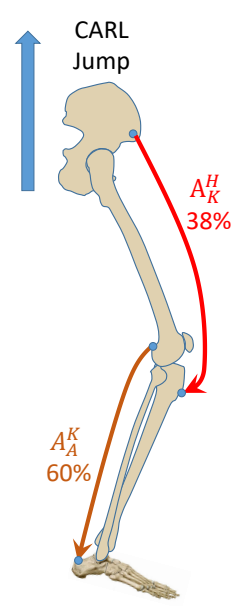

(a)

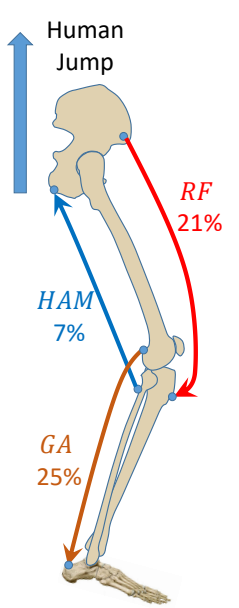

(b)

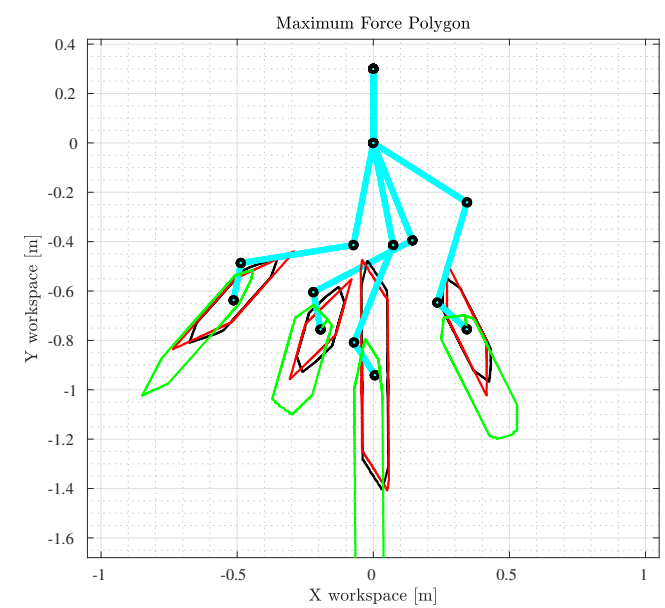

(c)

Fig. 4. (a) The power transfer capabilities of the BIAs during jump experiment with CARL. (b) For comparison, the energy transfer pattern of human vertical jump is depicted in the rightmost figure. The data is adopted from Ref. 12 The values are the percentage of the energy needed in the destination joint. For example, RF transfers $27 \%$ of the energy needed at the knee joint. (c) The maximum force polygon depicted for CARL actuator kinematics in Black Polygon. The red polygon belongs to the hypothetical mono-only configuration with actuators $50 \%$ stronger. The Green polygon is an estimation for the human leg muscle, moment arm data and muscle strength is taken from Ref. $13 \mid 14$

has to have higher moment arms to reach comparable joint torques.

In the human leg, the upper limb pseudo-antagonist biarticular muscles RF and HAM exhibit during various locomotion type an average hip-to-knee moment arm ratio of 0.7 and 2.0, respectively ${ }^{[10} \mathrm{CARL}$ is designed to have one upper limb biarticular $A_{K}^{H}$ acting as a compromise between function of RF and HAM with RMA of 1.0!10 The lower limb biarticular in humans is GA with an average ankle-to-knee moment arm ratio of 2.0, the similar ratio is adopted in the design of biarticular actuator $A_{A}^{K}$ in CARL. The moment arm of the BIAs are depicted in Fig. 3. The nonlinear MAP for BIAs is selected such that it exhibits the corresponding RMA during the joint trajectory of human walking, running and hopping! 10

\section{Discussion on Advantages and Disadvantages of Bi-articulation}

By augmenting a robotic arm or leg with redundant biarticular actuators, the designer attempts to capture the intended biological and technical advantages; however, as in any engineering problem, the addition of bi-articulation comes with its own challenging side-effects that have to be clarified. In this article, we mention the practical advantages of BIAs and provide insight on adverse technical issues that can discourage the adoption of BiAs in robotic systems.

The major motivation to adopt BIAs is to achieve a physiologically consistent function of the actuation system. It is hypothesized that the BIMs act as a ligament in which the muscle experiences relatively low contraction velocity while transferring power from upper limb strong muscles to lower limb joints. Power transfer is particularly important in movements with high power demand in the ankle joint like a squat jump. It is shown that in human vertical jump, $21 \%$ of energy needed in knee is transferred from hip joint via RF muscle ${ }^{\frac{15}{15}}$ see Fig. $4 \mathrm{~b}$. Similarly, $25 \%$ of work performed in the ankle joint is transferred from excess energy in the knee joint via 
GA $\stackrel{15}{15}$ This power transfer is essential for maximum jump movement since the ankle plantar flexor muscles (SOL and GA) alone do not have the strength to generate the required power in the ankle joint $\frac{15}{15}$ To replicate power transfer functionality in the robotic leg, actuation kinematics has to be designed such that BiAs experience low contraction/extension velocities during leg extension, acting similar to a ligament. The power transfer between joints can result in the higher mechanical efficiency of movement by decreasing the amount of negative power generated during motion.2

By replicating MAP of skeletal muscles in the actuation system of CARL, we have been able to achieve similar power transfer via biarticular actuators. By performing a vertical jump experiment $\frac{16}{16}$ it is demonstrated that upper limb biarticular $A_{K}^{H}$ has transferred $38 \%$ of energy required in the knee. Lower limb biarticular $A_{A}^{K}$ is able to transfer $60 \%$ of energy needed in the ankle joint, see Fig. 4a. In this particular jump experiment, the mechanical energy produced by actuators were 18\% less than the total energy (positive + negative energy) consumed in the joints. As a result, the mechanical efficiency of the jump movement is improved by $18 \%$ !10 Moreover, During explosive leg extension of the robotic jump experiment, the maximum contraction velocity of biarticular $A_{K}^{H}$ is $0.1 \mathrm{~m} \mathrm{~s}^{-1}$ which is 4 times less than contraction velocity of mono-articular at knee $A_{K}$ and 2 times less than hip mono-articular $A_{H}$, suggesting the bio-inspired ligamentous action of BIAs. These observations demonstrate that the efficiency achieved by the addition of biarticular actuators can indeed justify the extra added weight of redundant actuators in explosive movements.

BIAs are also studied based on their positive effect on the maximum force capability of the robotic arm and leg. A maximum force polygon is defined as the peak force that a robotic manipulator can generate around its end-effector in different directions. In Ref. [3, it is shown that the additional BIA can not only increase the size of the polygon but also reshape it in a way that the manipulator can generate uniform force around all the directions. The similar, design criterion is used in the design of the musculoskeletal robot Athlete $\frac{4}{4}$

The Maximum force polygon of CARL is shown in Fig. $4 \mathrm{c}$ in 4 different postures. The force polygon of the CARL, in black, is compared with a hypothetical mono-only configuration, in red color. The green polygon belongs to the nine-muscle-group abstraction of human leg presented in Fig. 1d. The MAP of the muscles are adopted from Twente lower extremity model (TLEM) 13 and the maximum muscle forces are adopted from Ref. 14. The maximum force of the muscles is decreased by $30 \%$ for ease of illustration of polygons. The asymmetric nature of the green polygon around the Ground Contact Point (GCP) is because the extensor muscles are stronger than their antagonists in the human leg.

In maximum force polygon corresponding to the mono-only configuration, it is assumed that BIAs are removed from CARL and instead the maximum force of MoAs is increased by $50 \%$, hence their weight is also increased by at least $50 \%$. By comparing the force polygons of CARL one belonging to mono-only configuration, it is apparent that there is a negligible difference between them. Indeed, the addition of biarticulars does not significantly improve the maximum force polygon of a conventional bipedal robot.

The hypothetical mono-only configuration would have much stronger actuators. The increase in the maximum force of the actuators can be achieved by either elongation of the moment arms or by increasing the gear ratio. The elongation of the moment arms normally is restricted by the mechanical structure since the compact design leaves small physical space to increase the moment arms. For instance, increasing the moment arm by $50 \%$ for $A_{K}$ actuator would change the length 
of the moment arm from $7 \mathrm{~cm}$ to $10.5 \mathrm{~cm}$, almost one-quarter of the link length, which in most robotic designs is not acceptable.

Alternatively, an increase in the gear ratio of the actuation system is equally not desirable since it deteriorates the force control performance of the actuators by increasing the reflected inertia of the rotor and worsening the friction and backlash effects. Indeed, an actuator with a higher gear ratio would not be desirably back-derivable and hence not suitable for impedance/force control. To alleviate such adverse transmission effects dual-motor SEA is proposed ${ }^{17}$ for wearable robotics, where two electric motors with different gear ratios are coupled via the planetary differential system. One motor is optimized for fast low power applications and the other one to meet high torque demand. It is shown that the efficiency of the actuator can be improved by $16 \%$.

In conclusion, it is reasonable to augment a robotic leg with additional BIAs rather than increase the gear ratio of the MoAs. Having extra BiAs not only is biologically sound but also improves the mechanical efficiency of the leg movement due to (a) power transfer of energy and (b) having back-drivable and efficient MoAs with lower gear ratio. The transmission of BIAs can be optimized for high torque low-velocity operations since BIAs experience significantly less contraction/extension velocities. On the other hand, MoAs can be optimized for high-velocity motions exhibited during the swing phase of locomotion.

One major problem in the mechanical design of linear BIAs is that their moment arms are relatively small compared to the other actuators. BiAs span two joints, for example, $A_{K}^{H}$ actuator spans hip joint with an angle range of $120^{\circ}$ and knee joint with a range of $90^{\circ}$. Each linear actuator has a limited extension length. The smaller the moment arm of the actuator is, the higher the range of the spanning joint angle. The moment arms of the BIAs have to be selected small so that BIAs can cover the whole motion range of two joints. This fact is easily observable in Fig. 3 where the moment arm of the BIAs are considerably shorter than corresponding muscles. In order to generate adequate torque, BIAs have to be selected with a higher gear ratio, which unfortunately is neglected in the design of CARL.

Although we acknowledged the practical advantages of the biarticular redundancy in the robotic leg, it is important to discuss the locomotion types in which the bi-articulation can be effective. Unfortunately, almost all the biomechanical and robotic studies, including ours, are highly focused on jumping and hopping movement. There is biomechanical evidence that in bouncing locomotion such as running, ${ }^{18}$ squat jump, ${ }^{12}$ BIMs can contribute to the efficiency of movements ${ }^{[19]}$ However, there is no clear evidence if the BIAs can improve the efficiency of walking in human. ${ }^{20}$ Indeed, RF muscle (upper limb biarticular) barely gets activated during walking. ${ }^{21}$ The activity of HAM muscle is also much lower compared to other locomotion modes. ${ }^{21}$ It is questionable if the integration of the upper limb biarticular actuator can be useful for the energetics of robotic walking.

Lower limb biarticular muscle GA has an activity pattern in synergy with ankle mono-articular SOL during walking. Despite a small difference in activity patterns of GA and SOL during catapult action, 22 it is fairly difficult to justify the existence of GA in a robotic system, since a one ankle MoA would be adequate to perform all the biological and technical roles in bipedal walking. Compared to running and jumping, the human walking motion is fairly static and therefore the power transfer capability of BIMs is not utilized.

Since most of the bipedal robots are designed specifically for walking locomotion the designer has to cautiously consider if the advantages of the BIAs can be utilized since their benefits can not be clarified in human locomotion. However, there is a technical benefit of using BiAs that 
can still be useful in walking bipedal robots. Bipedal robots, in general, suffer from positioning heavy actuators in the ankle joint due to the high torque/power demand. Using a lower limb biarticular actuator can be beneficial to transfer power from the upper knee joint to the lower ankle. Such a biarticular actuator can be positioned in the upper link thigh reducing the mass of the shank segment, as designed in LOLA robot.23

\section{Conclusion}

The objective of this article is to explain and demonstrate the practical advantages of redundant biarticular actuators in bipedal robotic systems. It was shown that the major physiological role of BIMs can be replicated in robotic systems to transfer power from heavy proximal segments to the lower limb joints. The BiAs can contribute to the efficiency of explosive movements in robotic systems similar to the human body. It is also discussed that despite the extra weight of redundant actuators, having redundancy can result in the customized selection of other MoAs in such a way that all the actuators operate in their optimum working points, additionally improving the efficiency of the robotic system. Although mounting biomechanical evidence suggests the benefits of the BIMs in explosive human movements, no specific advantage of BIMs is identified during walking locomotion. Therefore the functional advantages of BIAs can be limited for bipedal walking. At last, it is important to consider the technical advantage of the bi-articulation in facilitating the mass distribution of the actuators over leg segments. For instance, addition of lower limb biarticular can reduce the mass of the shank segment by placing the BIA on the thigh link.

\section{References}

1. K. Junius, M. Moltedo, P. Cherelle, C. Rodriguez-Guerrero, B. Vanderborght and D. Lefeber, Biarticular elements as a contributor to energy efficiency: biomechanical review and application in bio-inspired robotics Bioinspiration \& biomimetics 12, 2017.

2. G. J. V. I. Schenau, From rotation to translation: Constraints on multi-joint movements and the unique action of bi-articular muscles Human Movement Science 8, 1989.

3. V. Salvucci, Y. Kimura, S. Oh and Y. Hori, Force maximization of biarticularly actuated manipulators using infinity norm IEEE/ASME Transactions on Mechatronics 18, 2012.

4. R. Niiyama and Y. Kuniyoshi, Design principle based on maximum output force profile for a musculoskeletal robot Industrial Robot: An International Journal 37, 2010.

5. M. A. Sharbafi, C. Rode, D. Kurowski, R. Möckel, K. Radkhah, G. Zhao, A. M. Rashty and A. von Stryk, A new biarticular actuator design facilitates control of leg function in biobiped3 Bioinspiration E Biomimetics 11, 2016.

6. S. Schütz, K. Mianowski, C. Kötting, A. Nejadfard, M. Reichardt and K. Berns, RRLAB SEA A highly integrated compliant actuator with minimised reflected inertia, in IEEE International Conference on Advanced Intelligent Mechatronics (AIM), 2016.

7. S. Schütz, A. Nejadfard and K. Berns, Influence of loads and design parameters on the closedloop performance of series elastic actuators, in IEEE International Conference on Robotics and Biomimetics (ROBIO), 2016.

8. S. Schütz, A. Nejadfard, K. Mianowski, P. Vonwirth and K. Berns, CARL - A compliant robotic leg featuring mono- and biarticular actuation, in IEEE-RAS International Conference on Humanoid Robots, November 15-17, 2017.

9. A. Nejadfard, S. Schütz, P. Vonwirth, K. Mianowski and B. Karsten, Moment arm analysis of the biarticular actuators in compliant robotic leg carl, in Conference on Biomimetic and Biohybrid Systems, (Springer International Publishing, July 16-19, 2018).

10. A. Nejadfard, S. Schuetz, K. Mianowski, P. Vonwirth and K. Berns, Design of the musculoskeletal leg 
based on the physiology of mono-articular and biarticular muscles in the human leg Bioinspiration $\mathscr{E}$ biomimetics 14, 2019.

11. A. Hof, The force resulting from the action of mono-and biarticular muscles in a limb Journal of biomechanics 34, 2001.

12. R. Jacobs, M. F. Bobbert and G. J. van Ingen Schenau, Mechanical output from individual muscles during explosive leg extensions: the role of biarticular muscles Journal of biomechanics 29, 1996.

13. M. K. Horsman, The twente lower extremity model. consistent dynamic simulation of the human locomotor apparatus, PhD thesis, University of Twente12, 2007.

14. A. Rajagopal, C. L. Dembia, M. S. DeMers, D. D. Delp, J. L. Hicks and S. L. Delp, Full-body musculoskeletal model for muscle-driven simulation of human gait IEEE Transactions on Biomedical Engineering 63, 2016.

15. R. Jacobs and J. M. Macpherson, Two functional muscle groupings during postural equilibrium tasks in standing cats Journal of Neurophysiology 76, 1996.

16. A. Nejadfard, S. Schütz, P. Vonwirth, K. Mianowski and K. Berns, Coordination of the biarticular actuators based on instant power in an explosive jump experiment, in IEEE International Conference on Advanced Intelligent Mechatronics (AIM), July 9-12, 2018.

17. B. Vanderborght and D. Lefeber, A series elastic dual-motor actuator concept for wearable robotics, in Wearable Robotics: Challenges and Trends: Proceedings of the 4th International Symposium on Wearable Robotics, WeRob2018, October 16-20, 2018, Pisa, Italy, 2018.

18. R. Jacobs, M. F. Bobbert et al., Function of mono-and biarticular muscles in running. Medicine and science in sports and exercise 25, 1993.

19. B. I. Prilutsky and V. M. Zatsiorsky, Tendon action of two-joint muscles: transfer of mechanical energy between joints during jumping, landing, and running Journal of biomechanics 27, 1994.

20. B. I. Prilutsky, L. N. Petrova and L. M. Raitsin, Comparison of mechanical energy expenditure of joint moments and muscle forces during human locomotion Journal of biomechanics 29, 1996.

21. J. Perry, Gait Analysis: Normal and Pathological Function (SLACK, Thorofare, NJ, , 1992). OCLC: ocm27816876.

22. M. Ishikawa, P. V. Komi, M. J. Grey, V. Lepola and G.-P. Bruggemann, Muscle-tendon interaction and elastic energy usage in human walking Journal of applied physiology 99, 2005.

23. T. Buschmann, S. Lohmeier and H. Ulbrich, Humanoid robot Lola: Design and walking control Journal of Physiology Paris 103, 2009. 